UC-34a

Reporting Date: July 1974

Issued: August 1974

\title{
Thomas-Fermi Calculation of Potential Between Atoms
}

by

C. E. Lee

C. L. Longmire*

M. N. Rosenbluth **

*Permanent Address: Mission Research, Santa Barbara, CA.

* Permanent Address: Institute of Advanced Studies,

Princeton University, Princeton, NJ. 
This report was prepared as an account of work sponsored by the United States Government. Neither the United States nor the Unired States Atomic Energy Commission, nor any of their ernployees, nor any of their contractors, subcontractors, or their employees, makes any warranty, express or implied, or assumes any legal liability or responsibility for the accuracy, completeness or usefulness of any information, apparatus, product or process dis. closed, or represents that its use would not infringe privately owned rights.

In the interest of prompi distribution, this LAMS report was not edited by the Technical Information staff.

Printed in the United States of America. Available from National Technical Information Service

U.S. Department of Commerce 5285 Port Royal Road

Springfield, Virginia 22151

Price: Printed Copy $\$ 4.00$ Microfiche $\$ 1.45$ 


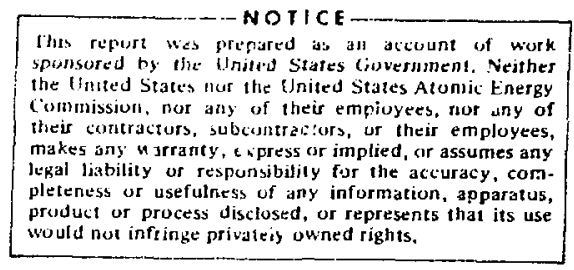

THOMAS-FERMI CALCULATION OF POTENTIAL BETWEEN ATOMS

by

C. E. Lee, C. L. Longmi re, and M. N. Rosenbluch

\section{ABSTRACT}

A Thumas-Fermi model calculation of the potential energy for the collision of two neutral, identical atoms is developed. The classical scattering distribution for this potential is compared to Rutherford scattering.

\section{INTROLUCTION}

In a collision between two atoms in which the atomic velocities are somewhat lower than the atomic electror velocities, on may expect the Thomas-Fermi model to give a fairly reasonable estimate of the interatomic force. Non-adiabatic effects such as the excitation of excited states and ionization will, of course, not come out of the calculation. Molecular binding effects will also not show up, for as we shall see, the Thomas-Fermi method can only give rise to a potential which is everywhere repulsive. The range of validity of such a calculation is therefore for atomic kinetic energies somewhat greater than, say, $10 \mathrm{eV}$ and for atomic velocities somewhat less than, say $10^{8} \mathrm{~cm} / \mathrm{sec}$.

In Sec. 1I, some general theorems conceming the Thomas-Fermi model are discussed. In Sec. III, a calculation of the potential energy for the collision of two neutral, identical atoms is described, and in Sec. IV, the classical scattering distribution for this potentiral is given.

II. GENERAL TIIEOREMS CONCERNING THE THOMHS-FL'RMI MOUEL

We imagine that the ion (nuclear) charge distribution $\rho_{+}$is held fixed by suitable extemal forces. The distribution $\rho_{+}$is a series of $\delta$-functions, but we need not specify it in detail at present, nor do we need to restrict our consideration to any specific number of nuclei. The Thomas-Fermi method then consists of solving the Poisson equation for the electrostatic potential $\phi$,

$$
\nabla^{2} \phi=-4 \pi \rho_{+}-4 \pi \rho_{-}
$$

where $\rho_{\text {, }}$ the electron charge density, is given by

$$
\rho_{-}=-A \phi^{3 / 2}
$$

Tie constant $\Lambda$ is given by

$$
A=\frac{2 e}{(2 \pi h)^{3}} \frac{4 \pi}{3}(2 m e)^{3 / 2} .
$$

Here the symbols have their usual meanings, and $\underline{e}$ is taken positive. According to the model, the kinetic energy $T_{\text {_ }}$ of the electrons is

$$
T_{-}=\frac{3}{5} A \int \phi^{5 / 2} d^{3} x
$$

We shall discuss three simple but interesting theorems that apply to the problem posed here. The first of these is the virial theorem, which is rather wej] known,* The virial $V$ is defined as usual as the integral of $\vec{r} \cdot \vec{F}(\vec{r}=$ position, $\vec{F}=$ force $)$ over the electron distribution,

$$
v=-\int \rho_{-} \overrightarrow{\mathbf{r}} \cdot \Gamma \phi d^{3} x
$$

*See P. Gombas, Die Statistische Theorie des Atons und ihre Anwendungen, Wien, Springer-Verlag, 1949. 
If the Thomas-Fermi expression (2) is used for $p$. here, one finds, after integration by parts, that

$$
V=-\frac{6}{5} A \int \phi^{5 / 2} d^{3} x=-2 T .
$$

On the other hand, if one replaces $\nabla \phi$ in $\mathrm{Eq},(5)$ by

$$
-\nabla \phi=\int \frac{\left(\vec{r}-\vec{r}^{\prime}\right)}{\left|\vec{r}-\vec{r}^{\prime}\right|^{3}} \rho\left(\vec{r}^{\prime}\right) d^{3} x ;
$$

one finds that

$$
v=v-\sum_{\text {ions }} \mathrm{ze}_{\mathrm{r}} \overrightarrow{\mathrm{r}} \overrightarrow{\mathrm{E}} .
$$

llere $V$ is the total electrostatic potential energy of electrons and iuns, and $\vec{E}$ is the electric field at position of the ion (excluding its self-field). The sum is extended over all ions. Comparison of Eqs. (6) and (7) yields the viral theorem

$$
V=-2 T_{-}+\sum_{\text {ions }} \text { ze } \vec{r} \cdot \vec{E} \text {. }
$$

The second theorem says that in any displacement of the ions, the work dune against the electric fields which they see goes into increasing $T_{-}+V-$ that is to say, $T_{-}+V$ is the potential energy for the motion of the ions. This theorem therefore amounts to the conservation of energy for the system of ions and electrons. To prove the theorem, we make a variation $\delta \rho_{+}$in the ion charge density. Through Eqs. (1) and (2), this will induce variations $\delta \phi$ and $S \rho_{-}$, which will satisfy

$$
\nabla^{2} \delta \phi=-4 \pi \delta \rho_{+}-4 \pi \delta \rho_{-}
$$

and

$$
\delta \rho_{-}=-\frac{3}{2} A \phi^{1 / 2} \delta \phi .
$$

Multiplying Eq. (1) by $\delta \phi$, Eq. (9) by $\phi$, subtracting and integrating over space yields $\left(\rho=\rho_{+}+\rho_{-}\right)$

$$
\int_{\phi \delta \rho_{+}}=\int \rho \delta \phi-\int_{\phi \delta \rho_{-}} .
$$

Now $\int \phi \delta p_{+}$is the work done by the ions against the electric fields which they see, and $\int \rho \varepsilon \phi$ is the change in the electrostatic potential energy of ions and electrons. Furthermore, - $\int \phi \delta \rho_{-}$is the work done by the elcctric field on the electrons, and this ought to equal the change in kinetic energy of the electrons. In fact, using Eq. (10),

$$
-\int \phi \delta \rho_{-}=\frac{3}{2} A \int \phi^{3 / 2} \delta \phi=\delta \mathrm{T}
$$

according to Fq. (4), and the theorem is proven.

The third theorem relates the electric forces on the ions to the electron pressure. According to the Maxwell stress theory, the electric force on the charges in a volume $V$ is related to the electric stresses on the surface $S$ of the volume,

$$
\int \rho E_{i} d^{3} x=\int n_{j} T_{i j} d^{2} x
$$

Here $E_{i}$ is the $i$ 'th component of the electric field, the vector $n_{j}$ is the inward normal to the surface, and $\mathrm{r}_{i j}$ is the electric stress rensor

$$
T_{i j}=\frac{1}{8 \pi} \delta_{i j} E^{2}-\frac{1}{4 \pi} E_{i} E_{j}
$$

in Cartesian coordinates. Separating $\rho$ into $\rho_{+}$and $\rho_{-}$, we find that the force on the ions in any volume is $\int_{V} p_{+} E_{i} d^{3} x=-\int p_{-} E_{i} d^{3} x+\int n_{j} T_{i j} a^{2} x$.

Now the electric force on the electrons is, according to $\mathrm{Eq}$. (2)

$$
\int_{V} \rho_{-} E_{i} d^{3} x=A \int_{V} \phi^{3 / 2} \frac{\partial}{\partial x_{i}} \phi d^{3} x=-\int p n_{i} d^{2} x
$$

where we have introduced the electron pressure

$$
p=\frac{2}{5} A \phi^{5 / 2}
$$

(Note that the pressure is two-thirds of the kinetic energy density, appropriate for an ideal gas.) Thus we may rewrite the electric force on the ions as

$$
\int \rho_{+} E_{i} d^{3} x=\int\left[p n_{i}+n_{j} T_{i j}\right] d^{2} x .
$$

This is the general form of the theorem. The utility of the theorem can be seen by applying it to a simple case where there are only two nuclei, which arc identical. If we choose the surface $S$ to be the plane which is the perpendicular bisector of the line joining the two nuclei, we find that the force on one nucleus is in a direction away from the other nucleus, and has magnitude 


$$
z e E=\int_{s}\left(p+\frac{r_{i}^{2}}{8 \pi}\right) d^{2} x .
$$

Since the integrand here is always positive, the force betwewn the two nuclei is always repuisive. Reduction to the simple form (1B) depended on the fact that $\vec{E}$ has wo component normal to the plane $S$, which is a result of symmetry in this case. In the general case, with several ron-identical nuclei, a similar simple form with positive total pressure holds for the surfaces which nowhere have any normal component of $\vec{E}$. It is therefore intuitively evident that no system of nuclei can be bound in this model.

III. CALCULATION OF TIIE POTENTINL FOR TWO IDENTICAL NEUTRAL ATOMS

For twe identical atoms, Eqs. (1) and (2) can be reduced to a universal set of equations independent of the nuclear charge $z$ by using the unit of length

$$
\lambda=(4 \pi \Lambda)^{-2 / 3} \mathrm{e}^{-i / 3} z^{-1 / 3}=0.885 \mathrm{a}_{0} / z^{1 / 3}
$$

where $a_{o}$ is the Bohr radius, and the unit of encrgy

$$
E=z^{2} e^{2} / \lambda
$$

The potential function $\phi$ is replaced by

$$
X=Z \mathrm{e} \phi / \varepsilon \text {. }
$$

With these units. Eqs. (1) and (2) become

$$
\nabla^{2} x=x^{3 / 2}
$$

with the boundary condition that

$$
x \sim\left|\vec{r}-\vec{r}_{n}\right|^{-1}
$$

near the position of each nucleus $\vec{r}_{n}$.

lquation (22) has been solved numerically on the IBM-704 for various distances between the two nuclei. Use was made of the solutions $x_{1}$ and $x_{2}$ for the two atoms separately; we let

$$
x=i_{1}+x_{2}+\psi
$$

so that

$$
\nabla^{2} \psi=\left(x_{1}+x_{2}+\psi\right)^{3 / 2}-x_{1}^{3 / 2}-x_{2}^{3 / 2} .
$$

This transformation removes the singularitics from $\psi$ at the positions of the nuclei, and probably improves the accuracy of the calculation when the nuclei are far apart. Instead of using Cartesian coordinates, we used the coordinates

$$
\begin{aligned}
& 2 r_{0}=\left|\vec{r}-\vec{r}_{1}\right|+\left|\vec{r}-\vec{r}_{2}\right| \\
& 2 \eta=\left|\vec{r}-\vec{r}_{1}\right|-\left|\vec{r}-\vec{r}_{2}\right| .
\end{aligned}
$$

In the differencing, cqual intervals of $n$ were used, and equal intervals of $\sqrt{\xi}$, the latter to reduce the number of points at large distancas from the nuclei where $X$ is small. For distances $\xi$ larger than about 12 times the separation of the nuclei, the solution was joined on to the Tromas-fermi solution for an atom with nuclear charge 22 .

The second-order Richardson relaxation scheme was used to arrise at the solution of Eq. (25). The kinetic and potential energy integrals $T_{-}+V$ werc then calculated, and the energies of the separated atoms $2 U_{0}$ subtracted, riving, the potential $U$ for the collision of the two atoms,

$$
u=T_{-}+V-2 u_{0} .
$$

The virial theorcm was used as a check on the accura cy. The third theorem of Sec. II, unfortunately, was not used as a thech. The virial check was such that we estimate that the calculated potential $U$ is accurate to ahout five nercent. The resules are given in Table 1 .

The asymptotic form of $1 /$ for ciose distances can be calculated. This asymptotic form is the potential energy of the two interactinp nuclei, $z^{2} c^{2} / d$, plus the difference in the binding energies of an atcon with nuclear charge $2 z$ and two atoms with nuclear charge 2 . The Thomas-Fcrmi binding cnerg is $-0.681 \varepsilon$, which is proportional to $2^{7 / 3}$. The asymptotic form of 11 is therefere, in our units

$$
\begin{aligned}
u & \rightarrow 1 / x=0.681\left(2^{7 / 3}-2\right) \\
& +1 / x=2.07
\end{aligned}
$$

*p. Combas, loc cit, p. G0 
TABLE 1

POTENTIAL ENERG AS A FUNCTION OF THE DISTANCE BETWEEN NUCLEI, IN UNITS GIVEN BY EQS. (19) AND (20).

\begin{tabular}{cc} 
Distance & Energy \\
\cline { 1 - 2 } $2 / 16$ & 14.4 \\
$1 / 8$ & 6.54 \\
$1 / 4$ & 2.77 \\
$1 / 2$ & 1.04 \\
1 & 0.318 \\
2 & 0.089 \\
4 & 0.0103
\end{tabular}

At a separation of $1 / 16$, this would give $U=13.97$, which disagrees with the entry in Table I by three percent.

We we re unable to determine the asymptotic form fo: large nuclear separation, but estimate from the pressure theorem of Sec. II that $U \sim \mathbf{r}^{-7}$ in this limit, using the known asymptotic form of the single atom runction. The numerical integrations obviously do not go this far out.

\section{CLASSICAL SCATTERING OF THO IDENTICAL NEUTRAL ATOMS}

The classical scattering theory becomes valid in general when the product of the wave number $k$ of the particle with the radius of the scattering potential -- the unit of leagth $\lambda$ in our case -- is very large compared to unity. Naking the approximation that the mass number of an atom is twice its $Z$, we find that the condition for the validity of classical scattering is

$$
k \lambda \approx 2 \times 10^{5} 2^{2 / 3}\left(\frac{v}{c}\right)>1
$$

where $v$ is the relative velocity of the atoms and $c$ is the velocity of light. For oxygen atoms, for example, $k \lambda$ is equal to thirty at an energy of about ten electron-volts, and the classical approximation should be quite good.

According to classical mechanics, for scattering in a certral potential $U(r)$, a particle with impact parameter $S$ is scattered through an angle $\theta$, in the center-of-mass system, given by

$$
\theta(s)=\pi-2 \int_{r_{1}}^{\infty} \frac{s d r}{r^{2} \sqrt{1-\frac{s^{2}}{r^{2}}-\frac{v}{i v}}}
$$

where $W$ is the center-of-mass energy, and $r_{1}$ is the value of $r$ at which the radical vanishes. Since only dimensionless ratios occur in Eq. (29), we may regard the units of length and energy to be the Thomas-Fermi units $\lambda$ and $\varepsilon$. The differential scattering cross section (per unit solid angle) in the center-of-mass system is

$$
\sigma(\theta)=\lambda^{2} \mathrm{~S} / \sin \theta \frac{\mathrm{d} \theta}{\mathrm{dS}} \mathrm{cm}^{2} \text { per steradian . (3U) }
$$

Equations (29) and (30) may be used to obtain $O(\theta)$ for various values of the energy parameter $w$. The result is naturally valid for all $z$ provided the length and energy units $\lambda$ and $E$ are cmployed. rigure (1) shows $\sigma(e) / \lambda^{2}$ for various energies. It is seen that for small energies, where the potential lnoks "hard," the scattering is more isotropic than at high energies, where it iends towards the Rutherford shape. It should be noted that if the incident and struck particles are not distinguished, the effective scattering cross section is the sun of $\sigma(\theta)$ and $J(\pi-\theta)$.

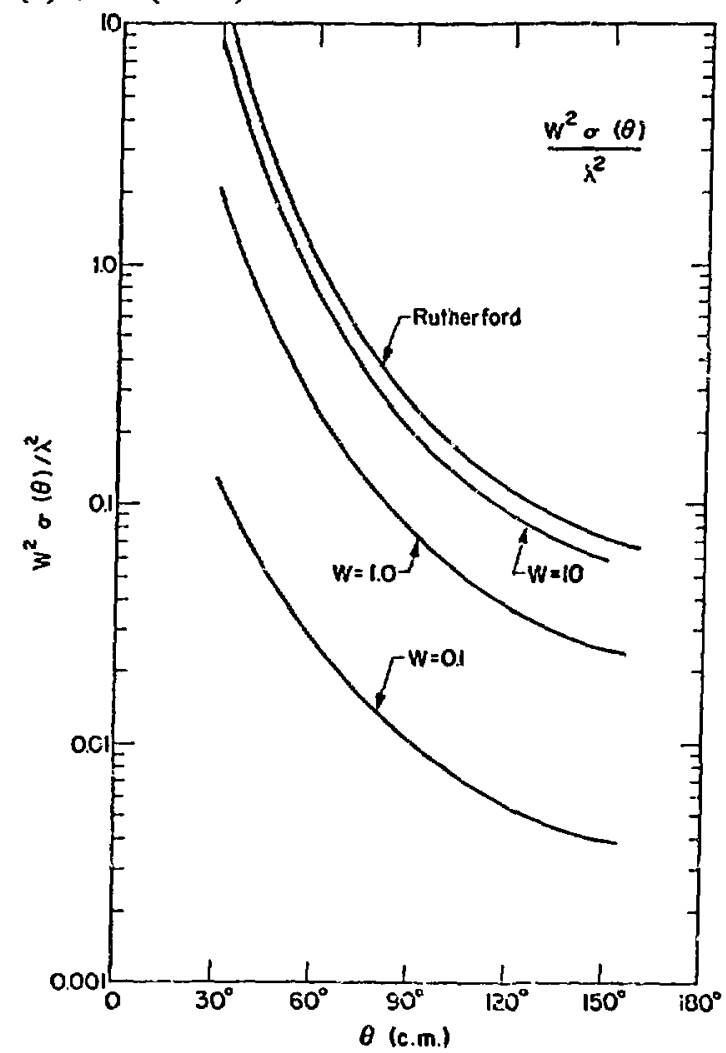

EE: $268(130)$
Figure 1 